\title{
The Effect of Endurance Training on Apoptotic Inducing Factor (AIF) in Cerebellum of Type 1 Diabetic Rats
}

\author{
${ }^{1}$ Asma Taheri, ${ }^{1}$ Abdolhamid Habibi*, ${ }^{1}$ Saeed Shakeryan, ${ }^{1}$ Masoud Nikbakht, \\ ${ }^{2,3}$ Mohammad Reza Tabandeh \\ ${ }^{1}$ Department of Sport Physiology, Faculty of Sport Sciences, Shahid Chamran University of Ahvaz, Ahvaz, \\ Iran. ${ }^{2}$ Department of Basic Sciences, Division of Biochemistry and Molecular Biology, Faculty of \\ Veterinary Medicine, Shahid Chamran University of Ahvaz, Ahvaz, Iran. ${ }^{3}$ Stem Cells and Transgenic \\ Technology Research Center, Shahid Chamran University of Ahvaz, Ahvaz, Iran.
}

Submitted 27 January 2019; Accepted in final form 20 March 2019.

\begin{abstract}
Background. The apoptotic inducing factor (AIF) level increases in the brain of patients with diabetic disease. Objectives. This study aimed to investigate whether endurance training could alleviate apoptosis in the cerebellum of diabetic rats. Methods. Twenty rats were randomly allocated in four groups including 1) healthy control, 2) healthy trained, 3) diabetic, and 4) diabetic trained groups. Diabetes was induced by intraperitoneal injection a single dose of Streptozotocin $(45 \mathrm{mg} / \mathrm{kg})$. Training programs on the treadmill were performed at moderate intensity 5 days a week for 6 weeks. The activities apoptotic inducing factor was analyzed in cerebellum of animals at the end of experiment by ELISA method. Results. Our results showed that following six weeks endurance exercise training decreased cytosolic release of apoptotic inducing factor (AIF) in cerebellum of diabetic rats. Conclusion. Our results confirmed that endurance training may be improves brain functions. Understanding mechanisms of exercise on brain functions may lead to the development of novel therapeutic strategy for neurodegenerative disorders such as type 1 diabetes.
\end{abstract}

KEYWORDS: Endurance Training, Apoptotic Inducing Factor, Type 1 Diabetes, Rat.

\section{INTRODUCTION}

Diabetes is a type of metabolic disease that causes functional changes in the nervous that has been increasing in the last decades $(1,2)$. Autonomic and peripheral neuropathy are well known complications in diabetic patients (3). There is increasing evidence that diabetes condition is associated with some forms of neuropathy. Insulin signaling in the brain regulates neuronal survival, synaptic plasticity and cognitive and locomotor functions (4). One particular brain region where high expression of insulin receptor has been found is the cerebellar cortex (5). Previous studies in humans and experimental animals have shown that locomotor abnormality, cognition impairment and sensory discrimination in diabetic patients may be due to molecular and pathological changes in cerebellum (6). Ultrastructural changes and an increase in apoptotic activity have been reported in cerebellum of the type 1 diabetic rats (7).

Apoptosis is well-coordinated and strictly controlled process in which cytotoxic insults result in cellular proteolysis and DNA fragmentation. In the intrinsic pathway of apoptosis, pro apoptotic signals such as the proapoptotic Bcl-2 family proteins $\mathrm{Bax}$ and $\mathrm{Bak}$ )

*. Corresponding Author:

Abdolhamid Habibi, Professor

E-mail: hamidhabibi330@gmail.com 
translocate to the mitochondria, resulting in mitochondrial membrane premeabilization. This in turn provides as route for release of intermembrane space proteins such as cytochrome $\mathrm{c}$ and apoptosis inducing factor (AIF) into the cytosol that promotes the formation of the apoptosome and cell death. $(8,9)$. Atrophy of the cerebellum has been reported in diabetic patients, and this is not associated with the duration of the disease or glycemic control $(10,11)$. Increasing evidence has shown that exercise can be a powerful tool to reduce the symptoms of neuropathy, in particular, cognitive impairment and abnormal motor function in various neurological disorders. It is now clear that optional exercise can stimulate neurogenesis, increase resistance to brain failure, and improve learning and mental function in diabetic patients (12). Endurance exercise training can prevent the onset the natural history of diabetic peripheral neuropathy (13).

According to best of our knowledge, there is little evidence about the potential protective effect of endurance exercise against oxidative stress and apoptosis in the cerebellum of diabetic animal. Therefore the present study was aimed to investigate whether endurance training could alleviate apoptosis in the cerebellum of streptozotocin (STZ) induced diabetic rats.

\section{MATERIALS AND METHODS}

Experimental Animals and Grouping. In this experimental study, twenty healthy adult male Wistar rats $(200-220 \mathrm{~g})$ were obtained from the experimental animal holding of Shahid Chamran University of Ahavz, Ahvaz, Iran. The animals were housed in standard cages and maintained under controlled room temperature $\left(23 \pm 1^{\circ} \mathrm{C}\right)$ and $55 \pm 4 \%$ humidity with $12 / 12 \mathrm{~h}$ light/dark cycle. They were provided rat chow (Pars, Tehran, Iran) and water at libitum. All animals used were cared for according to the guide for the care and use of laboratory animals by the national academy of sciences (National Institutes of Health publication No. 86 - 23). All rats were housed in conventional conditions and fed standard diet and water ad libitum at the animal facility for 1 week before experiments began. Animals were allocated to the following groups: (1) normal control group, in which diabetes was not induced (CON; $n=5)$, (2) normal exercise group, in which diabetes was not induced but the rats were subjected to treadmill exercise $(\mathrm{TH} ; \mathrm{n}=5) ;(3)$ diabetes control group, in which diabetes was induced but the rats were not subjected to exercise
$(\mathrm{DC} ; \mathrm{n}=5$ ) and (4) diabetes exercise group, in which diabetes was induced and the rats were subjected to treadmill exercise (TD; $\mathrm{n}=5$ ). Animals in DC and TD groups were injected a single intraperitoneal injection of $50 \mathrm{mg} / \mathrm{kg} \mathrm{STZ}$ dissolved in $0.9 \% \mathrm{NaCl}$ after $12 \mathrm{~h}$ fasting. On the second day after injection, all injected rats were checked for the induction of diabetes by assessment of blood glucose levels by tail prick using hand held glucometer (EasyGluco, Mainland, China). Animals with fasting blood glucose levels above $250 \mathrm{mg} / \mathrm{dL}$ were accepted as diabetic. The CON and $\mathrm{TH}$ groups received only a saline injection. After diabetes induction exercise training was began. All experimental protocols were approved by an ethics committee of Shahid Chamran University of Ahvaz for animal and human experiments (EE/97.24.3.69971/scu.ac.ir) and were in accordance with international ethical standards (14).

Exercise Training Protocols. The rats in the TH and TD were subjected to moderate intensity treadmill exercise 5 days per week for 6 weeks. The speed and duration of the treadmill exercise were gradually increased from $10 \mathrm{~m} / \mathrm{min}$ for 10 min (grade $0 \%$ ) in the 1 st week to $10 \mathrm{~m} / \mathrm{min}$ for 20 min (grade 0\%) in the 2nd week, $14-15$ $\mathrm{m} / \mathrm{min}$ for $20 \mathrm{~min}$ (grade $0 \%$ ) in the 3rd week, 14 - $15 \mathrm{~m} / \mathrm{min}$ for $30 \mathrm{~min}$ (grade $0 \%$ ) in the 4 th week, and $17-18 \mathrm{~m} / \mathrm{min}$ for $30 \mathrm{~min}$ (grade $0 \%$ ) from the 5th week. Exercise stayed constant as same as week 5 for last week to obtain the given adaptations under steady state condition.

Tissue Sampling. At the end of experiment, all animals were sacrificed under ether anesthesia. They were decapitated; their cerebellums were removed with dissection and tissues were frozen in $-70^{\circ} \mathrm{C}$ for subsequent analysis.

Evaluation of Cytosolic Level of AIF. To obtain cytosolic protein, tissues were minced and gently homogenized with 4 volumes of ice cold lysis buffer containing $0.25 \mathrm{M}$ sucrose, $10 \mathrm{mM}$ Tris- $\mathrm{HCl}$, $\mathrm{pH} 7.4,1 \mathrm{mM}$ EDTA, and $250 \mu \mathrm{g} / \mathrm{ml}$ bovine serum albumin. Protease Inhibitor Cocktail (Sigma, St. Louis, MO, USA) were added to the samples. Samples where then centrifuged at $14,000 \times \mathrm{g}$ for 15 min at $4^{\circ} \mathrm{C}$. Supernatants, containing predominantly soluble protein, were stored at $-80^{\circ} \mathrm{C}$ until ELISA analysis. Protein concentration of the supernatant was estimated using the Bradford technique. AIF concentration was measured using rat specific ELISA kit (MyBioscience, USA) and expressed as $\mathrm{ng} / \mathrm{mg}$ protein. The limit of detection of AIF was $0.05 \mathrm{ng} / \mathrm{ml}$, and the intra-assay and interassay 
coefficients of variation were less than $10 \%$ and $11 \%$, respectively.

Statistical Analysis. All statistical surveys were performed using SPSS version 20 software (SPSS Inc., Chicago, IL, USA). Data was presented as mean \pm SEM. Initially, the normal distribution of data and the homogeneity of variances were performed using Shapiro-Wilk tests and Leven was evaluated. One way analysis of variance (ANOVA) and Tukey post hoc test were applied for multiple comparisons of different factors between groups. The level of significance for all tests was set at $\mathrm{p}<0.05$.
Effect of Endurance Training on Blood Glucose Level. Blood glucose level was significantly increased $48 \mathrm{~h}$ after STZ injection in DC and TD groups $(\mathrm{p}<0.05)$ (Figure 1). Endurance training had no significant effects on blood glucose concentration in TD groups between 1 to 4 weeks after diabetes induction $(\mathrm{p}<0.05)$, while it caused reduction of blood glucose level at 5 th and 6 th weeks of experiment compared with DC groups $(\mathrm{p}<0.05)$ (Figure 1). Exercise program had no obvious effects on blood serum of healthy animals during the experiments $(\mathrm{p}<0.05)$ (Figure 1).

\section{RESULTS}

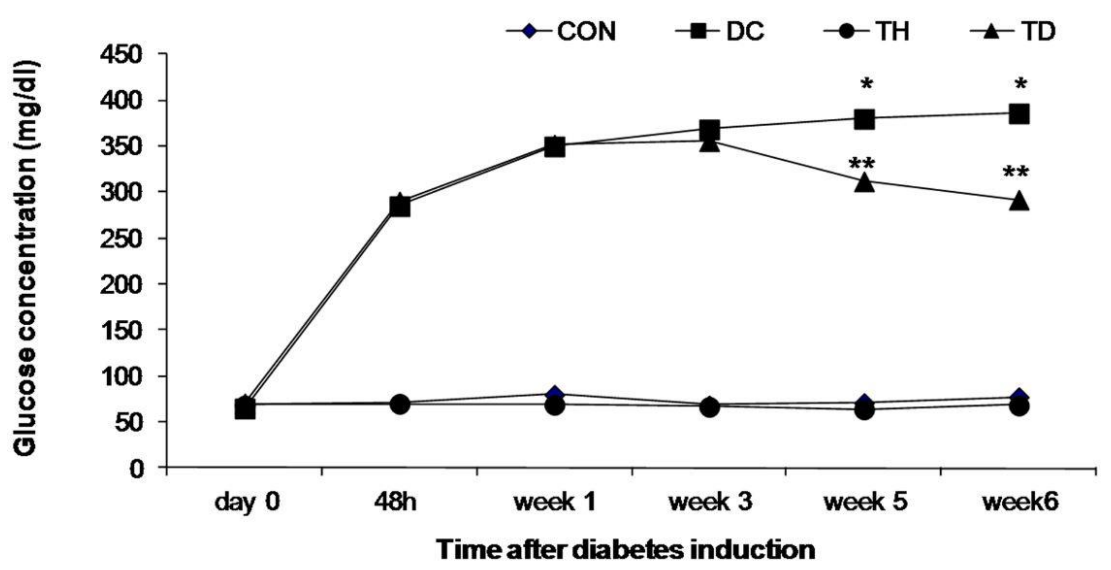

Figure 1. The alteration of blood glucose concentration in different experimental groups before and after diabetes induction. Data was obtained from 10 animals in each group at each studied time. Values are presented as mean \pm SEM. Different number of * show significant difference at p<0.05. CON: healthy group; DC: untrained, diabetic group; TH: healthy, trained group; TD: diabetic, trained group.

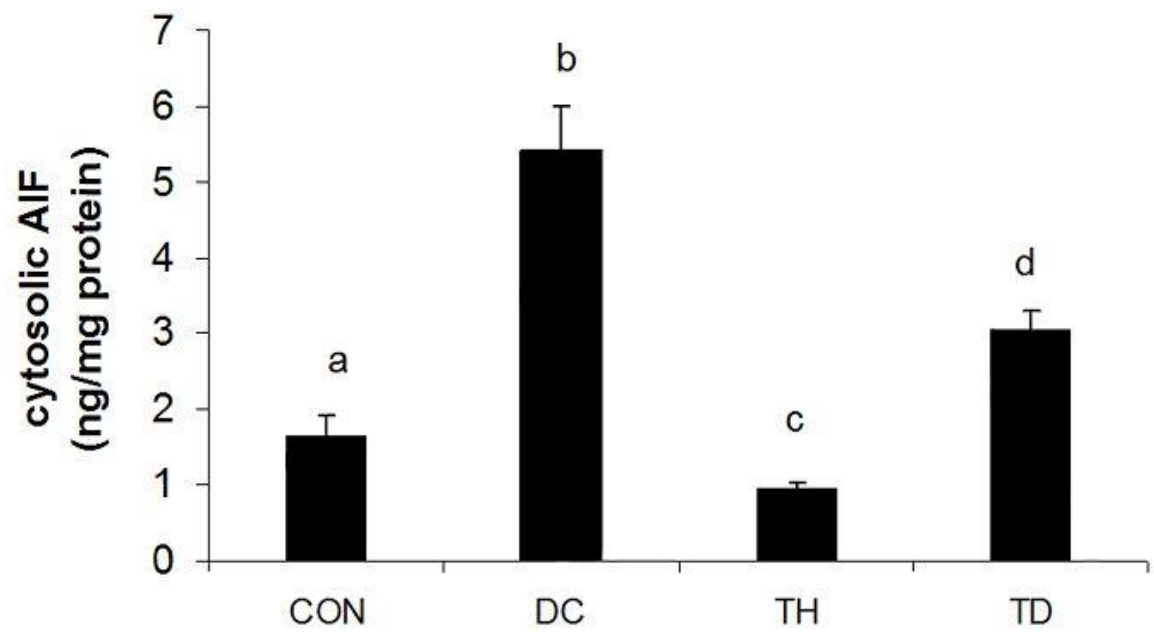

Figure 2. Cytosolic level of apoptotic inducing factor (AIF) in cerebellum of different experimental animals. The level of each studied factor was normalized relative to total tissue protein concentration and expressed as $\mathrm{ng} / \mathrm{mg}$ protein. Data was obtained from 10 animals in each group. Values are presented as mean \pm SEM. Different letters demonstrate significant difference between groups at $p<0.05$. CON: healthy group; DC: untrained, diabetic group; TH: healthy, trained group; TD: diabetic, trained group. 


\section{Effect of Endurance Training on Release AIF into the Cytosol}

A significant elevation of cytoplasmic level and AIF was observed in cerebellum of diabetic rats 6 weeks following diabetes induction $(\mathrm{p}<0.05)$ (Figure 2). Our results showed that the levels of both protein was decreased in cerebellum of diabetic rats following 6 weeks endurance training $(\mathrm{p}<0.05)$ (Figure 2). Trained, healthy animals showed lower level AIF concentrations compared with healthy, untrained animals $(\mathrm{p}<0.05)$ (Figure 2).

\section{DISCUSSION}

Diabetes slows brain activity and reduces mental flexibility and can interfere with daily functioning and have adverse effects on the quality of life of diabetic patients (15). In addition, in diabetic conditions, hyperglycemic stress causes oxidative damage to mitochondria, which may lead to apoptosis and cell death $(16,17)$. However, few studies have explained the alteration of cerebellar cellular mechanisms following aerobic exercise $(18,19)$. So far, little is known about the protective effect of endurance exercise on the apoptosis process in the cerebellum of diabetic rats. In the present study, we investigated the possible protective effects of endurance exercise on apoptosis induced in the cerebellum by hyperglycemia in STZ-type diabetic rats.

In our study, diabetic animals had higher blood glucose compared with control group. The level of glucose in the diabetic animals was reduced following 6 weeks endurance training, suggesting that the prescribed exercise regimen resulted in a reduction of blood glucose after inducing diabetes. Although the training protocol improved the blood glucose in diabetic animals, it did not able to return blood glucose to normal level. These results reflect the chronic adaptations induced by exercise training in diabetic patients that has been reported in previous study (18). Apoptosis confirmed by detection of increased AIF proteins in cerebellum of diabetic rats. The releasing of AIF; as final effector of apoptosis reduced in trained, diabetic animals when compared with untrained, diabetic rats. Although endurance training had beneficial effects against diabetes induced apoptosis in cerebellum, it could not return the levels of studied factors to normal values, confirming that exercise can only use as a complementary approach to attenuate the adverse effects of hyperglycemia in cerebellum. Based on our knowledge data on cerebellar changes at molecular levels in diabetic condition are more limited and less studied $(18,20)$. Previous works have confirmed the presence of mitochondrial dysfunction and oxidative stress in different regions of brain in diabetic animal model that can trigger the apoptosis and cell death.

One of the main contributions of the present study was to show for the first time that the apoptosis related molecular events promoted in the cerebellum of rats by hyperglycemia can be minimized following endurance physical exercise. In our study, we found that endurance exercise could reduce the release of AIF into the cytosol in cerebellum of diabetic animals. Several studies have shown that bcl 2 protein family members are important components of the apoptosis process that regulate mitochondrial outer membrane permeability and release of proapoptotic factors, such as cytochrome $\mathrm{c}$ and AIF from mitochondria, and this may be a mechanism It is important for diabetes related to neurological disorders (8).

Excess glucose for insulin-dependent tissues such as the brain can increase mitochondrial and glycolysis metabolic pathways and NADH accumulation in mitochondria and oxidative stress (21). New evidence confirms the indirect regulation of mitochondrial biogenesis by contributing to AIF and oxidative phosphorylation system, and acts as an antioxidant when entrapped in the mitochondrial (6). Reduction of $\mathrm{NAD}^{+}$in diabetic conditions balances the inactive monomeric form of AIF, which can pass through mitochondrial membrane openings, release into the cytosol and then move to the nucleus, where it contribute to lethal processes such as chromatinolysis. (22). Recently, research has shown that exercise can balance the $\mathrm{NAD}^{+} / \mathrm{NADH}$ ratio in mitochondria and prevent $\mathrm{NAD}^{+}$accumulation through various mechanisms such as activation of $\mathrm{NAD}^{+}$ mitochondrial cycles, PPAR related $\mathrm{O} 2$ consumption and uncoupling of ATP synthesis, NAM phosphoribosyl transferase (23). It is likely that exercise prevents the accumulation of $\mathrm{NAD}^{+}$ and subsequent apoptotic events by reducing blood glucose levels and glucose uptake by the brain. To confirm this opinion, recent research by Honkala et al. (24) showed that Aerobic exercise can reduce brain glucose uptake in different areas of the cerebral cortex. Further study are necessary to determine the molecular mechanism of endurance training on attenuation of release or expression of apoptotic factors in cerebellum in diabetes condition. 


\section{CONCLUSION}

This study provides the first evidence that six weeks endurance exercise training attenuate apoptosis events in cerebellum of diabetic rats. Our results confirmed the role of aerobic exercise in improving cerebellum molecular disturbances in diabetic patients that may lead to the development of novel therapeutic strategy for improvement of diabetes neuropathy. Further studies are needed to identify the best time to start aerobic exercise after diabetes incidence and the best exercise protocol with various duration and intensity to obtain the best effects.

\section{APPLICABLE REMARKS}

According to the results of this study, people are recommended to use endurance exercise training to improve cerebellum molecular disturbances in diabetic patients that may lead to the development of novel therapeutic strategy for improvement of diabetes neuropathy.

\section{ACKNOWLEDGMENT}

This work was funded by a Grant from Shahid Chamran University of Ahvaz Research Council (Grant No: 97/3/02/16670).

\section{AUTHORS' CONTRIBUTIONS}

All authors contributed to the study conception and design. Abdolhamid Habibi, Mohammad Reza Tabandeh, Saeed Shakeryan and Masoud Nikbakht designed the study. Asma Taheri performed the study and collected the data. Abdolhamid Habibi, Saeed Shakeryan and Mohammad Reza Tabandeh analyzed the results. Mohammad Reza Tabandeh and Asma Taheri drafted the article. Asma Taheri and Abdolhamid Habibi revised the article critically for important intellectual content. All authors gave final approval of the version to be published. Mohammad Reza Tabandeh is the guarantor of this work and, as such, had full access to all the data in the study and takes responsibility for the integrity of the data and the accuracy of the data analysis.

\section{CONFLICT OF INTEREST}

The authors declare that there is no conflict of interest.

\section{ETHICAL APPROVAL}

The conduct and procedures involving animal experiments were approved by the Shahid Chamran University Committee for Ethics in Animal Experiments (License number: EE/98.24.3.26209/SCU.AC.IR) in compliance with the guideline for the care and use of laboratory animals (NIH publication no. 86-23). They were adapted for 7 days before the starting of the experiment.

\section{REFERENCES}

1. Guariguata L, Linnenkamp U, Beagley J, Whiting DR, Cho NH. Global estimates of the prevalence of hyperglycaemia in pregnancy. Diabet Res Clin Pract. 2014;103(2):176-185. doi: 10.1016/j.diabres.2013.11.003 pmid: 24300020

2. Kalalian-Moghaddam H, Baluchnejadmojarad T, Roghani M, Goshadrou F, Ronaghi A. Hippocampal synaptic plasticity restoration and anti-apoptotic effect underlie berberine improvement of learning and memory in streptozotocin-diabetic rats. Eur $J$ Pharmacol. 2013;698(1-3):259-266. doi: 10.1016/j.ejphar.2012.10.020 pmid: 23099256

3. Niyomchan A, Sricharoenvej S, Lanlua P, Baimai S. Cerebellar Synaptopathy in Streptozotocin-Induced Diabetic Rats. Int J Morphol. 2019;37(1).

4. Cotman CW, Berchtold NC. Exercise: a behavioral intervention to enhance brain health and plasticity. Trend Neurosci. 2002;25(6):295-301.

5. Kleinridders A, Ferris HA, Cai W, Kahn CR. Insulin action in brain regulates systemic metabolism and brain function. Diabet. 2014;63(7):2232-2243. doi: 10.2337/db14-0568 pmid: 24931034

6. Klein JA, Longo-Guess CM, Rossmann MP, Seburn KL, Hurd RE, Frankel WN, et al. The harlequin mouse mutation downregulates apoptosis-inducing factor. Nature. 2002;419(6905):367-374. doi: 10.1038/nature01034 pmid: 12353028

7. Hernandez-Fonseca JP, Rincon J, Pedreanez A, Viera N, Arcaya JL, Carrizo E, et al. Structural and ultrastructural analysis of cerebral cortex, cerebellum, and hypothalamus from diabetic rats. Exp Diabetes Res. 2009;2009:329632. doi: 10.1155/2009/329632 pmid: 19812703

8. Lechuga-Sancho AM, Arroba AI, Frago LM, Paneda C, Garcia-Caceres C, Delgado Rubin de Celix A, et al. Activation of the intrinsic cell death pathway, increased apoptosis and modulation of astrocytes in 
the cerebellum of diabetic rats. Neurobiol Dis. 2006;23(2):290-299. doi: 10.1016/j.nbd.2006.03.001 pmid: 16753303

9. Razavi SM, Abdollahi M, Salamati P. Cancer events after acute or chronic exposure to sulfur mustard: A review of the literature. Int J Prev Med. 2016;7:76. doi: 10.4103/2008-7802.182733 pmid: 27280012

10.Lunetta M, Damanti AR, Fabbri G, Lombardo M, Di Mauro M, Mughini L. Evidence by magnetic resonance imaging of cerebral alterations of atrophy type in young insulin-dependent diabetic patients. J Endocrinol Invest. 1994;17(4):241-245. doi: 10.1007/BF03348967 pmid: 7930374

11. Ghasemi M, Behnaz F, Hajian H. The Effect of Dexmedetomidine Prescription on Shivering during Operation in the Spinal Anesthesia Procedures of Selective Orthopedic Surgery of the Lower Limb in Addicted Patients. Anesth Pain Med. 2018;8(2):e63230. doi: 10.5812/aapm.63230 pmid: 30009149

12. Berchtold NC, Castello N, Cotman CW. Exercise and time-dependent benefits to learning and memory. Neurosci. 2010;167(3):588-597. doi: 10.1016/j.neuroscience.2010.02.050 pmid: 20219647

13. Dixit S, Maiya AG, Shastry BA. Effect of aerobic exercise on peripheral nerve functions of population with diabetic peripheral neuropathy in type 2 diabetes: a single blind, parallel group randomized controlled trial. J Diabet Complicat. 2014;28(3):332-339. doi: 10.1016/j.jdiacomp.2013.12.006 pmid: 24507164

14. Harriss DJ, MacSween A, Atkinson G. Ethical standards in sport and exercise science research: 2020 update. Int J Sport Med. 2019;40(13):813-817. doi: 10.1055/a-1015-3123 pmid: 31614381

15. Mijnhout GS, Scheltens P, Diamant M, Biessels GJ, Wessels AM, Simsek S, et al. Diabetic encephalopathy: A concept in need of a definition. Diabetolog. 2006;49(6):1447-1448. doi: 10.1007/s00125-006-0221-8 pmid: 16598451

16. Bo H, Jiang N, Ji LL, Zhang Y. Mitochondrial redox metabolism in aging: Effect of exercise interventions. J Sport Health Sci. 2013;2(2):67-74.

17. Muriach M, Flores-Bellver M, Romero FJ, Barcia JM. Diabetes and the brain: oxidative stress, inflammation, and autophagy. Oxid Med Cell Longev. 2014;2014:102158. doi: 10.1155/2014/102158 pmid: 25215171

18. Borges ME, Ribeiro AM, Pauli JR, Arantes LM, Luciano E, de Moura LP, et al. Cerebellar insulin/IGF1 signaling in diabetic rats: Effects of exercise training. Neurosci Lett. 2017;639:157-161. doi: 10.1016/j.neulet.2016.12.059 pmid: 28034783

19.JalalTaherabadi S. Effect of endurance training on cerebellar gene expression of the ADP-ribosylation factor 6 in rats with diabetic peripheral neuropathy. Zahedan J Res Med Sci.20(12).

20. Cardoso S, Santos RX, Correia SC, Carvalho C, Santos MS, Baldeiras I, et al. Insulin-induced recurrent hypoglycemia exacerbates diabetic brain mitochondrial dysfunction and oxidative imbalance. Neurobiol Dis. 2013;49:1-12. doi: 10.1016/j.nbd.2012.08.008 pmid: 22940631

21. Wu J, Jin Z, Zheng H, Yan LJ. Sources and implications of NADH/NAD(+) redox imbalance in diabetes and its complications. Diabet Metab Syndr Obes. 2016;9:145-153. doi: 10.2147/DMSO.S106087 pmid: 27274295

22. Cheung EC, Joza N, Steenaart NA, McClellan KA, Neuspiel M, McNamara S, et al. Dissociating the dual roles of apoptosis-inducing factor in maintaining mitochondrial structure and apoptosis. EMBO J. 2006;25(17):4061-4073. doi: 10.1038/sj.emboj.7601276 pmid: 16917506

23. White AT, Schenk S. NAD(+)/NADH and skeletal muscle mitochondrial adaptations to exercise. Am J Physiol Endocrinol Metab. 2012;303(3):E308-321. doi: 10.1152/ajpendo.00054.2012 pmid: 22436696

24.Honkala SM, Johansson J, Motiani KK, Eskelinen JJ, Virtanen KA, Loyttyniemi E, et al. Short-term interval training alters brain glucose metabolism in subjects with insulin resistance. J Cereb Blood Flow Metab. 2018;38(10):1828-1838. doi: 10.1177/0271678X17734998 pmid: 28959911 